\title{
Dry needling of the gastrocnemius muscle. Overlap between trigger point area and the presence of blood vessels
}

\author{
Velasco Fernández P. ${ }^{1}$ Valera Garrido F. ${ }^{1}$ \\ ${ }^{1}$ Sannus Clinic Medical and Sports Center, Pozuelo de Alarcón, \\ Madrid, Spain
}

Rev Fisioter Invasiva 2019;2:63.

\begin{abstract}
\section{Keywords}

- venous system

- lower limb

- gastrocnemius

- anatomic variations

- dry needling

- myofascial pain syndrome

Aims To evaluate the anatomic variability of the gastrocnemius venous system using ultrasound and the overlap of the same with myofascial trigger points (MTrPs 1 and 2) clinically located in this muscle.

Material and Methods In total, 82 legs of 41 healthy adults were studied, belonging to 26 men and 15 women aged between 22 and 50 years. The blood vessels located below MTrP1 and MTrP2, described by Travell and Simons were observed, and each image was analyzed by sectors (medial, central and lateral) to quantify the number of blood vessels, their distribution and overlap with the MTrPs described at this level.

Results Examination of the 164 heads of 82 gastrocnemius muscles revealed that at least one vessel exists for each section analyzed. The number of veins per head varied between 1 and 8 . The most common pattern in the medial gastrocnemius was three vessels $(41.5 \%)$ and two vessels (49\%) in the lateral gastrocnemius. In $100 \%$ of the cases the localization of the veins coincided with the clinical localization of the MTrPs. Conclusions The venous pattern of the proximal gastrocnemius is highly variable among subjects regarding the number of blood vessels and their distribution. There is a complete overlap between the clinical localization of the gastrocnemius MTrPs and the presence of blood vessels.
\end{abstract}

